\section{Quantitative In Situ Mechanical Testing in Electron Microscopes}

\author{
M. Legros, D.S. Gianola, and C. Motz
}

about the surface (e.g., optical and SEM) but have some advantages with regard to temporal resolution over 3D probing technologies, which can require long acquisition times to enhance the signal quality. Figure 1 is a three-axis map of the main in situ tools used to investigate deformation mechanisms. The first two axes are the strain resolution and the length scale, which rely on the size of the probe, the incoming wavelength, and the signalto-noise ratios of the sensors and imaging devices. The third axis is the time resolution, which, in part, is dependent on the detectors. The spatial resolution of optical in situ microscopy and Raman is limited by their wavelength and can be improved by looking at ensembles of small objects. $X$-ray in situ studies require a very intense and focused beam to investigate small deforming volumes in short amounts of time. Such experiments can only be performed at modern synchrotron sources offering high brightness and advanced detectors; the expense of these experiments precludes repeated tests. ${ }^{1,2}$

SEM and TEM are clearly well adapted for micron- and submicron-sized specimens. The lower time limit is often due to the speed of detectors or video frame rates. Recently, this limit has been extended to ultra-fast dynamic imaging by using lasertriggered beams and synchronous detection. In the case of electron microscopy, this very specific tool, for instance, is currently employed to study fast chemical reaction phase transformations ${ }^{3}$ (for a review, see Reference 4) but will not be discussed here. In this article, we focus our attention on in situ electron microscopy studies where the time intervals are on

\section{Introduction}

In situ transmission electron microscopy (TEM) and scanning electron microscopy (SEM) have long proved to be effective tools to investigate intrinsic deformation mechanisms as they unfold. Slip band formation, dislocation motion and interactions, and crack nucleation and propagation are examples where direct observations gleaned significant insight. With significant technological advances in high-resolution and fast digital imaging, microactuators, and highfidelity sensors, SEM and TEM have clearly opened new horizons toward dynamically acquiring quantitative data during in situ experiments. A parallel driving force for the development of in situ electron microscopy is the miniaturization of functional building blocks (micro- and nanoelectromechanical systems [MEMS, NEMS], submicron interconnects, nanowires, thin films, micropillars) down to sizes that are similar to TEM and SEM samples. These objects exhibit unusual mechanical properties in comparison to their bulk counterparts, which causes us to question our current understanding of their modes of deformation at and below the micron scale. Acquiring knowledge of the physical basis leading to such structural alterations in addition to quantitative data about these changes has therefore become of paramount importance, scientifically and technologically.

Several imaging platforms across a large range of length scales are appealing for in situ mechanical testing. Conventional imaging systems only provide information

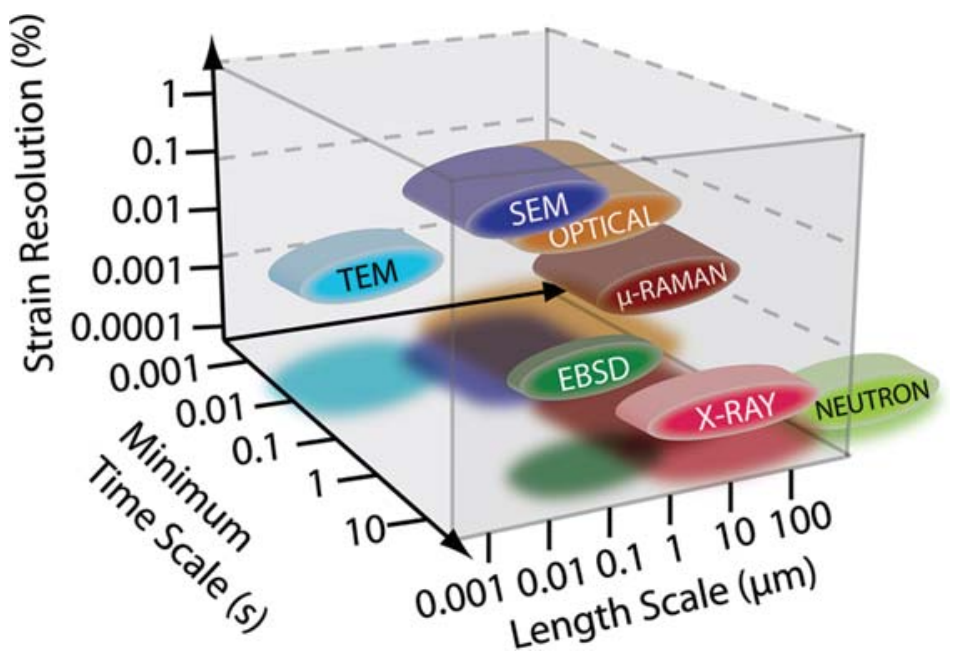

Figure 1. Common in situ methods mapped schematically to illustrate their ability to extract quantitative strain information as a function of minimum time and length scales. EBSD, electron back-scattered diffraction. 
the order of standard video frame rates ( 30 frames per second).

\section{Scanning Electron and Focused- Ion-Beam Microscopies}

The combination of in situ mechanical testing using scanning electron and focused-ion-beam (FIB) microscopies has recently sparked a number of studies, 5,6 although first reports date back to the late 1960s. Gane and Bowden reported in situ indentation, compression, and bending in SEM and TEM.7,8 Dingley later demonstrated in situ tensile and compressive testing in SEM. ${ }^{9}$ In situ SEM approaches are generally very versatile in terms of the diversity of specimens (since electron transparency is not required), instrumentation, and environments that can be studied. Commercial vendors of both standalone (SEM or FIB) and dual-beam (SEM and FIB) configurations now offer large chamber designs and modular configurations of auxiliary components such as gas-injection systems, nanomanipulators, and sensors to allow for flexible multi-use laboratories that are ideally suited for in situ investigations. The versatility of these dual-beam machines offers some advantages over in situ TEM approaches, where the specimen and stage volume is limited. First reports of detailed tests on micrometersized samples inspired by strain gradient plasticity formulations were performed in torsion and bending in the 1990s, 10,11 where the typical specimen sizes were in the range of several tens of microns. Recently, similar microbending tests were scaled down to specimens with sizes of a few micrometers and smaller. ${ }^{12-14}$ In the Examples/Case Studies section of this article, examples are given of microbending and nanotensile tests that take advantage of the combination of FIB milling, actuation, and force detection capabilities offered by recent FIB/SEM-based systems.

\section{Transmission Electron Microscopy}

In situ TEM testing is experimentally more delicate: the space available between the pole pieces is generally less than one centimeter. Samples have to be integrated in the microscope by a specific sample holder that performs the desired operations, where all the instruments and coupling to the specimen must be fit into small volumes. The most common in situ TEM holders for mechanical testing are room-temperature (RT) tensile straining holders. Some holders are capable of cooling or heating, although most do not provide quantitative data output. These were developed soon after the advent of TEM (e.g., the first heating stage was reported by Silcox and Whelan in
196015). Those stages are now commercially available. Straining holders capable of working at low or elevated temperatures are often custom made by individual laboratories to accommodate specific configurations. ${ }^{16,17}$ The use of such simple holders has led to considerable insight regarding dislocation-based plasticity in semiconductors, ${ }^{18,19}$ quasicrystals, ${ }^{20}$ metals, alloys, ${ }^{21-24}$ and metallic thin films. ${ }^{25,26}$ In fact, in situ TEM is the sole technique capable of directly measuring dislocation mobilities at the nanoscale because of its ability to see projections of the interior of the sample and visualize individual dislocation mechanisms. ${ }^{27}$ Even when plastic deformation is carried out by alternative means such as grain-boundary (GB) shear coupling, the quantitative and dynamical data that are drawn from in situ TEM straining experiments are unique. An example of such an experiment performed on small-grained $\mathrm{Al}$ is given in the Examples/Case Studies section of this article.

\section{Experimental Setup/Testing Techniques and Approaches Specimen Preparation-Selection}

Micron- and submicron-scale specimens can either be fabricated from bulk or larger size components (top-down) or grown to a size (bottom-up) that can be handled by holders and micromanipulators. The classical example of a top-down specimen preparation is FIB-fabricated pillars that can be fashioned from virtually any bulk specimen and tested in compression using a flatpunch nanoindenter. ${ }^{28}$ This approach has sparked a number of studies and questioned the traditional laws of plasticity at small scales.2,29-32 Overall, the FIB technique has become most prominent for the preparation of micrometer-sized specimens, as it allows one to mill almost any material in a variety of geometries and sizes (several 100 nm up to a few tens of microns) (see Figure 2). However, FIB preparation has recently been shown to introduce a large number of defects near the surface ${ }^{33}$ and to have dramatic effects on the mechanical response in the case of Mo-based pillars. ${ }^{34,35}$ Current studies, therefore, are trying to understand the possible effect of FIB milling or to circumvent the FIB step to investigate size effects on mechanical behavior. A recent approach is the differential chemical etching of eutectic alloys that leads to pillars of similar sizes but introduces limited damage. ${ }^{36}$ These objects tend to behave mechanically like whiskers as they reach the theoretical strength of the crystal, independent of their size.

Bottom-up methods (e.g., vapor-liquidsolid nanowire growth, templated electrodeposition) appear as interesting alternatives to explore size effects in mechanical behavior, particularly on very small samples $(<200$ $\mathrm{nm})$. For instance, metallic nanowhiskers produced by near-equilibrium growth using physical vapor deposition in an ultrahigh vacuum system combine a near-perfect crystalline lattice and a constant diameter ( 10-500 $\mathrm{nm}$ ) over the entire length (defined by the growth crystallography), making them strong candidates for bending or uniaxial testing. ${ }^{37,38,63}$

Similarly, TEM specimens can be classified as either objects that are fabricated from bulk materials or those that are already thin enough to be tested in their as-fabricated state. However, all of them are required to be, at least locally, electron transparent and therefore exist in thin foil configurations. This thin foil geometry has excluded most tests apart from tensile thus far, but the emergence of nanowires and small FIBmade specimens render bending tests possible (Figure 2d), where compressive stress states could locally be attained. ${ }^{39} \mathrm{New}$ nanoindenter-based TEM holders (see next section) can also implement such compressive states, but the sample geometry has to be adapted, as shown in Reference 40. In this article, examples are given of two $\mathrm{Al}$ samples strained in tension. One is a single crystal deposited on polyimide that has been shaped with the FIB on the polymer side, minimizing irradiation effects. The second one is an ultrafine grained $\mathrm{Al}$ bulk sample prepared by electrochemical polishing, a classic technique for metals that does not substantially modify the structure of the specimen. More information is available on MEMS approaches that are ideal for in situ studies. $^{41}$

\section{Actuation, Force, and Deformation Measurements}

To perform in situ TEM straining in tension, the displacement has to be transmitted to the sample in a very accurate and rigid way. Any elastic energy stored within the transmission line between the actuator and the sample can lead to rapid failure of fragile specimens. In addition, most crystalline materials thinned down to a few hundreds of $\mathrm{nm}$ in the strained zone become intrinsically fragile. Using micromotors and rigid shafts, in situ tensile holders can typically apply displacement rates ranging from $10 \mathrm{~nm}$ to $1 \mu \mathrm{m}$ per second. Many experiments also are performed by applying small increments of deformation and observing relaxation events. The required rigidity of the strain application mechanism renders the introduction of a load cell between the sample and the actuator very difficult, although careful and well-designed experiments have been recently successful. ${ }^{42-44}$ 
a

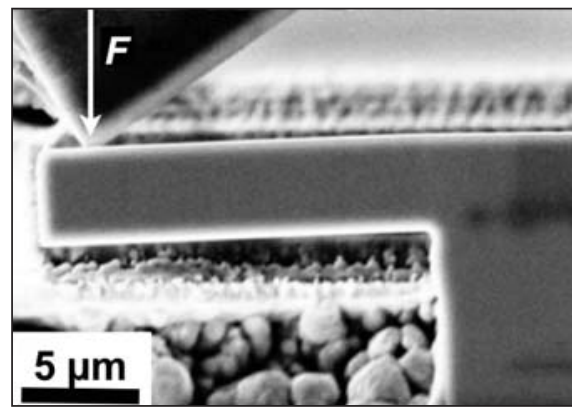

C

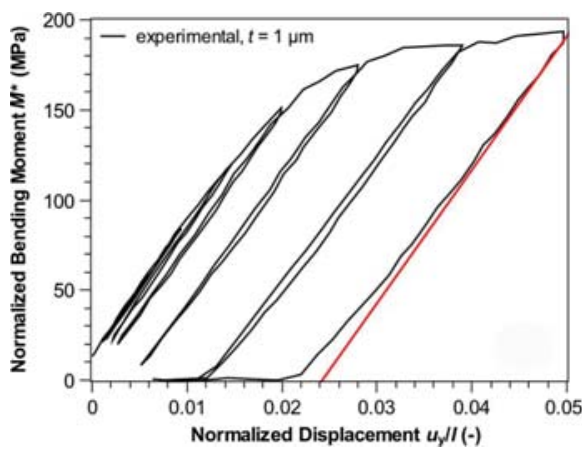

$\mathrm{b}$

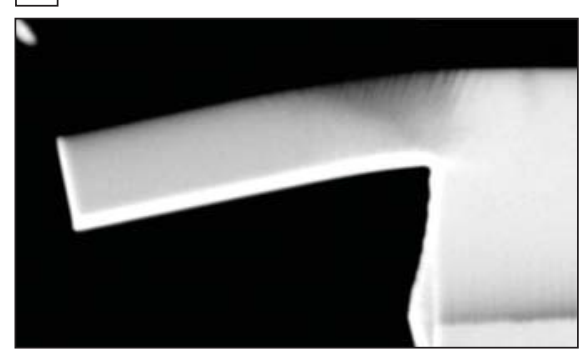

d

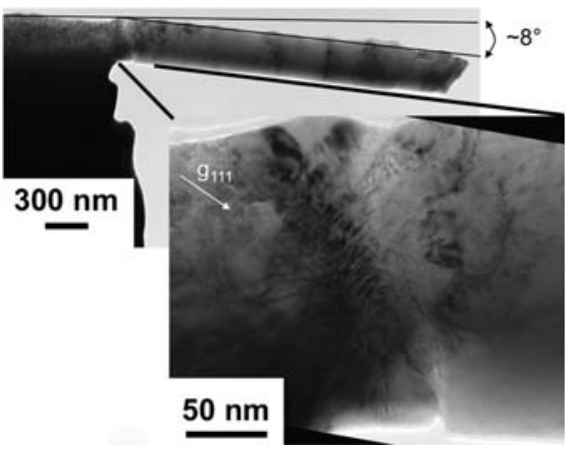

Figure 2. Example of the in situ loading of bending beams. (a) Experimental scanning electron microscopy setup showing side views of the 5 - $\mu \mathrm{m}$-thick bending beam and indenter; $F$ is force. (b) Back-scattered electron micrograph of a heavily deformed beam. (c) Plot of the moment versus transverse displacement, $u_{y}$ (normalized by the beam length, I) for a 1- $\mu \mathrm{m}$-thick beam with several unloading/loading sequences. The red curve represents a linear unloading slope to demonstrate that nonlinearity sets in before reaching zero stress (from the positive direction). This is indicative of early-stage Baushinger effect. (d) Example of an in situ transmission electron microscopy bending experiment of a $\mathrm{Cu}$ beam.

In the specific case of thin films attached to rigid substrates, a heating or cooling holder can serve to plastically deform the film by exploiting the difference in coefficient of thermal expansion (CTE) between the metal film and the substrate..$^{39,45}$ This difference in CTE produces small but controllable strains. ${ }^{46}$ This also serves as an actuation mechanism in certain MEMS devices developed for in situ TEM. ${ }^{47}$

A more versatile option relies on piezoelectric actuators that provide subnanometer motion. Integrated capacitive, strain gauge, or optical sensing technologies that allow for closed-loop operation can ameliorate issues such as DC drift and hysteresis that typically afflict piezoelectric materials. This fine displacement can be combined either with micrometers to ensure a large range of motion of the sample or with other piezo units that can work in stick-slip mode (also known as inertial drive stages). The latter approach has been successfully used as the underlying technology for nanomanipulators and positioners in FIB-SEM machines but has only recently been adopted to TEM holders. Another example of a high fidelity force application and measurement system that can be miniaturized to fit in a TEM holder employs electrostatic force actuators and three-plate capacitive displacement sensing.

These new technical developments have catalyzed a number of studies on nanometer-scaled objects that were not possible previously, mostly involving compression of pillars. ${ }^{33,48,49}$ Measuring the applied load also benefited from the miniaturization of load cells, and those can now be inserted in an actuator-sample-sensor series, even in TEM sample holders cited previously. Capacitive sensors are generally used for larger forces $(\mu N)$, while atomic force microscopy-type cantilevers are employed for the smaller ones $(\mathrm{nN})$. The range of possibilities of these holders for applications in in situ mechanical testing is currently being explored.

\section{Image-Based Measurements Methods of Strain and Stress}

Real-time surface morphology evolution during concurrent applied deforma- tion can be digitally captured during testing. For instance, crack density or surface deformation can be calculated using image processing tools such as digital image correlation (DIC) and tracking. DIC computes sub-pixel displacements and strains from intensity changes between reference and deformed images but requires a relatively stable contrast between consecutive images and good pixel-wise signal-to-noise ratio. SEM and FIB images offer such conditions, but low noise images typically require slower scan times that could introduce significant drift or stress relaxation during image acquisition. Nevertheless, the variety of electron detection schemes can highlight different sources of contrast (secondary, backscattered electrons) depending on the material being studied. Sutton and colleagues recently reviewed some of the challenges and opportunities for using DIC in a SEM imaging environment. ${ }^{50,51}$ Their work details the steps necessary for conducting spatially resolved full-field strain measurements with relatively high precision ( 0.02 pixel resolution, which corresponds to $\sim 25 \mathrm{~nm}$ at $200 \times$ magnification and $\sim 0.5$ $\mathrm{nm}$ at $10,000 \times$, and a strain resolution of $\left.\sim 1.5 \times 10^{-4}\right)$. We will see here how the DIC technique has been applied to calculate local strains developing in nanometer-sized $\mathrm{Cu}$ whiskers and micron-sized $\mathrm{Cu}$ beams.

Although the automated DIC technique as applied to TEM images has been heretofore elusive, video stills are now sufficiently sharp to perform manual image calculation after in situ TEM experiments, either to calculate dislocation velocity ${ }^{27}$ or GB shear coupling. ${ }^{52,53}$ Because of the remarkable sensitivity of the electron/crystal interaction, several image-based stress measurement techniques are available for TEM. The simplest one consists of measuring the curvature of mobile dislocations. A fully identified (Burgers vector $\boldsymbol{b}$, slip plane), curved dislocation provides a good indication of the local stress as computed via the simplified equation:

$$
\tau=\frac{\mu b}{R}
$$

where $\mu$ is the shear modulus, $R$ is the radius of curvature, $\tau$ is the local stress resolved in the glide plane, and $b$ is the magnitude of the Burgers vector. This method is the only one that directly measures a stress. When no dislocations are present, stress measurement is often based on strain detection that can have picometer resolution, such as the recent dark field holography or highresolution TEM techniques coupled with geometrical phase analysis developed by Hÿtch et al. ${ }^{54,55}$ The stress is then back calculated based on classical elasticity. 


\section{Examples/Case Studies: Plastic Deformation of Confined Volumes Single-Crystal Deformation with Finite-to-Large Dislocation Densities}

Measurements of strengths in both bcc and fcc single-crystalline micro- and nanopillars by compression give values systematically well above bulk ones. ${ }^{56}$ Yet, they generally fall short of reaching the ideal strength. In fact, microwhiskers synthesized by the reduction of halides demonstrated tensile strengths systematically higher than pillar data for a given size, as reported 50 years ago by Brenner. ${ }^{57}$ Similarly, Bei and colleagues recently reported ideal strengths of dislocation-free Mo-alloy pillars around the micron range. ${ }^{36,58}$ Given estimates of dislocation densities (and accordingly, mean dislocation spacing) in the well-annealed single crystals being tested, this discrepancy between measured and theoretical strengths of FIB-fabricated pillars is surprising, since the smallest pillars have a finite probability of being dislocation-free. However, in situ TEM compression of $\mathrm{Ni}$ pillars showed that large amounts of preexisting defects are present due to FIB damage. ${ }^{33}$ This damage was concentrated near the surface and was driven out of the crystal upon the application of small amounts of external load (termed "mechanical annealing"), leaving a visibly defect-free crystal requiring the nucleation of dislocations to proceed with plastic deformation. Yet the subsequent tests do not reproduce whisker-like values of stress. In fact, Norfleet and co-workers demonstrated that the idea of sampling a given dislocation density when carving a pillar is erroneous, as this density was reported to increase with decreasing pillar size. ${ }^{59}$ The discrepancy between pillar and whiskers behavior raises the question of whether one or several mechanisms (dislocation source truncation, dislocation starvation, strain gradient effects) is responsible for the enhanced strength of small objects.

\section{Single Crystal Bending}

Single crystal bending can partially test the validity of these proposed mechanisms, since bending requires the permanent storage of geometrically necessary dislocations to accommodate the strain gradient. Figure 2a illustrates the principle of an in situ bending SEM test performed on $\mathrm{Cu}$ beams: the indenter tip is applied to the extremity of the beam in displacement control. The specimens were prepared from a bulk copper single crystal with an $<110>\{111\}$ orientation using FIB milling. ${ }^{13}$ Typical sizes for the specimens were 1 to $8 \mu \mathrm{m}$ in thickness, 2.5 to
$10 \mu \mathrm{m}$ in width, and 10 to $35 \mu \mathrm{m}$ in length During loading, images of the deformed region were taken with different detectors to identify the deformation patterns (Figure $2 a$ and $2 \mathrm{~b}$ ), as well as the force versus displacement response (Figure 2c). These types of tests can also be extended to in situ TEM investigations to identify active dislocation processes, which are illustrated in Figure 2d.

In this test, a sign reversal of the stress and strain occurs at the neutral axis, represented by the dashed line in Figure 3b. Dislocation loops, nucleated near the surfaces where the stresses are highest, expand toward the free top or bottom surface and also into the bulk. By moving toward the neutral axis of the beam, the driving force (resolved shear stress) on the dislocations decreases and reaches zero at the neutral axis. Dislocations, therefore, may stop at the neutral axis, but pileups may push the leading dislocations over this point. However, negative resolved stress after the neutral axis favors the creation of dislocation pileup around the neutral axis (Figure $3 b$ ). Such dislocation pileups induce long-range stress fields that act as back stresses on the active dislocation sources, provoking their immobilization. This mechanism was supported by in situ experimental observations of slip traces extending only from the surface to the center of the beam and by 3D discrete dislocation dynamics simulations. ${ }^{60}$ This additional contribution to the size effect is larger in thinner beams and contrasts with uniaxial testing, where slip traces extend over the whole cross-section. A strong Bauschinger effect also has been evidenced for thinner beams, which manifests as asymmetry between the yield strengths in tension and compression and is caused by the dissolution of pileups during unloading.

The size dependence of the normalized bending moment (proportional to average flow stress) that scales approximately

\section{a}
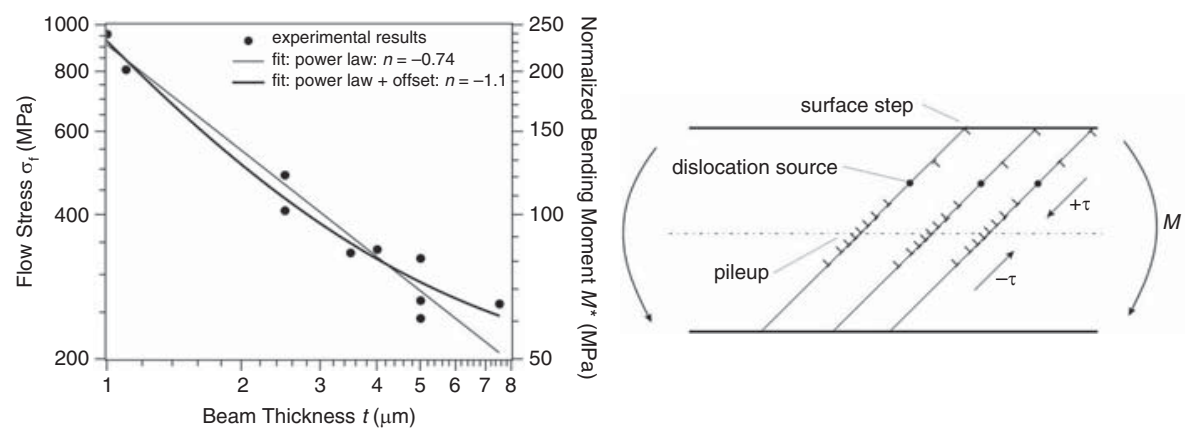

Figure 3. (a) Size dependence of flow stress and normalized bending moment, respectively, on the beam thickness; (b) assumed dislocation structure inside the beam causing the pronounced size effect. $M$ is the applied bending moment, and $\tau$ is the shear stress on the glide plane.

inversely with the beam thickness (Figure 3a) is more pronounced than for microcompression and tension tests. This "complex loading" case study shows that under specific loading conditions of confined volumes, dislocation structures can build up and govern the mechanical properties. As shown by early works of Fleck et al., ${ }^{10}$ strain gradients are accommodated by geometrically necessary dislocations, which contribute to the total dislocation density $\rho_{\text {tot' }}$ especially if the strain gradients are high. According to the Taylor relation:

$$
\sigma_{\mathrm{f}} \propto \sqrt{\rho_{\text {tot }}}
$$

the flow stress $\sigma_{\mathrm{f}}$ will follow the dislocation density. At the macroscopic scale, these gradients are usually very small, and individual dislocation processes can be safely neglected. At the micro- or nanometer regime, individual dislocation processes and arrangements strongly affect the mechanical properties and therefore should be accurately monitored.

\section{Single-Crystal Deformation with Low Dislocation Density}

In contrast to what was shown in submicron pillar compression, ${ }^{33}$ a tensile test on a similarly-sized Al single crystal strained in situ in the TEM to more than $140 \%$ did not show any evidence of dislocation starvation, a phenomenon associated with the facile annihilation of dislocations at free surfaces. In the following experiment, a single-crystalline $\mathrm{Al}$ film supported by a thin $(8 \mu \mathrm{m})$ polyimide substrate was thinned down to electron transparency ${ }^{61}$ and line-patterned along the TEM holder straining axis (Figure 4a). The early failure of the polyimide at the notch (Figure $4 b-4 c$ ) may be attributed to the irradiation. This process left a bare

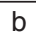


a

\section{FIB (FIB image)}

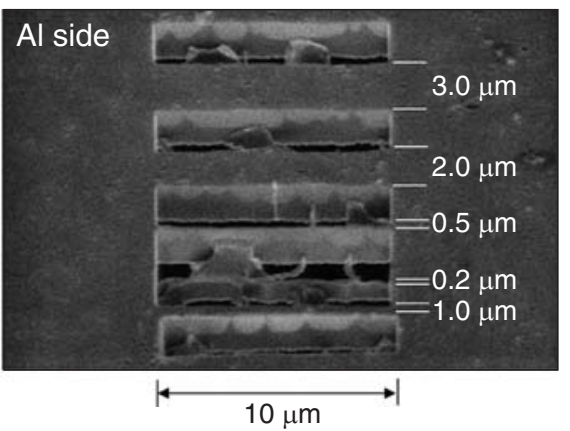

$\mathrm{b}$

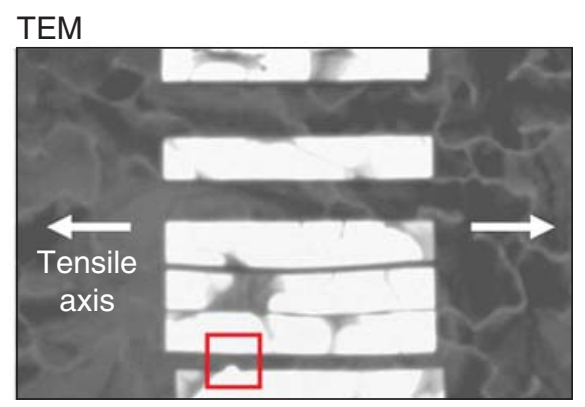

C

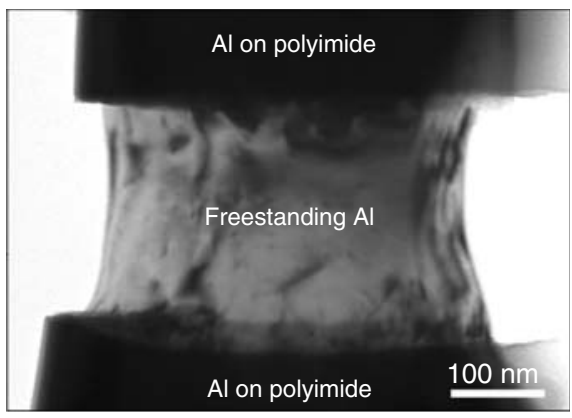

Figure 4. In situ transmission electron microscopy (TEM) straining of a submicron Al ligament obtained by focused-ion-beam patterning of a 400-nm-thick Al film deposited on polyimide. The polyimide substrate is first milled down to $2 \mu \mathrm{m}$ to create a $250 \mu \mathrm{m}^{2}$ wide zone that is transparent to electrons. The second patterning, also performed from the polyimide side, is visible in (a) ionic imaging and (b) TEM imaging and consists of long $\mathrm{Al} /$ polyimide ligaments with various widths. One ligament was notched (red square) to concentrate the deformation. At this exact location, the in situ tensile testing causes a split of the polyimide substrate, leaving bare a single crystalline Al ligament (c), whose $\langle 100\rangle$ axis is perfectly aligned with the tensile axis, vertical in (c). ${ }^{62}$ 400-nm-wide Al crystal, perfectly aligned for multiple slip condition (Figure 4c). The in situ TEM experiment precisely measures the strain between the two edges of the fractured polyimide substrate, and, at the same time, observations of dislocation nucleation (spiral sources) and their escape through the free surfaces of the crystal were collected. Since minimal strain gradients are expected here, the dislocations are not expected to accumulate in the crystal. In fact, for a constant strain rate, the dislocation density remained constant at about $3 \times 10^{10} \mathrm{~cm}^{-2} .{ }^{62}$

\section{Whisker Behavior (Dislocation-Free)}

In the previous example, local stress could be estimated using dislocation curvature. In defect-free nano-sized whiskers $(<300 \mathrm{~nm})$, this approach is not possible. To perform clean, readily interpretable nanomechanical experiments at length

a

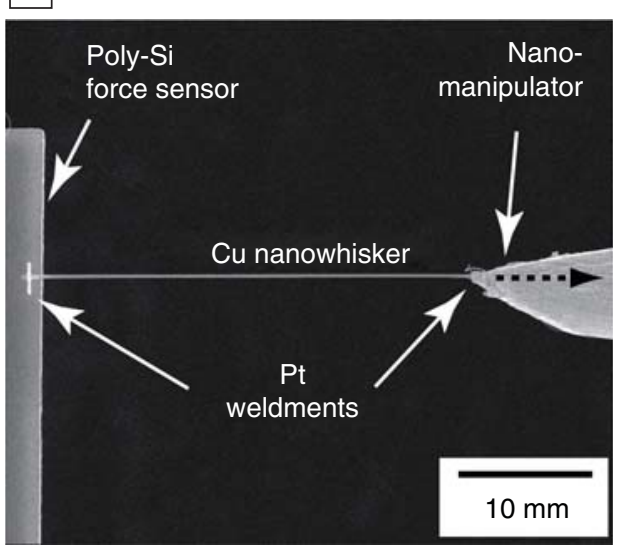

$\mathrm{b}$

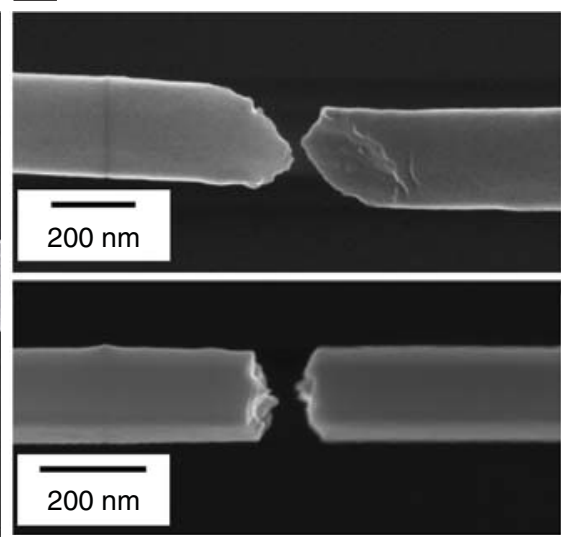

c
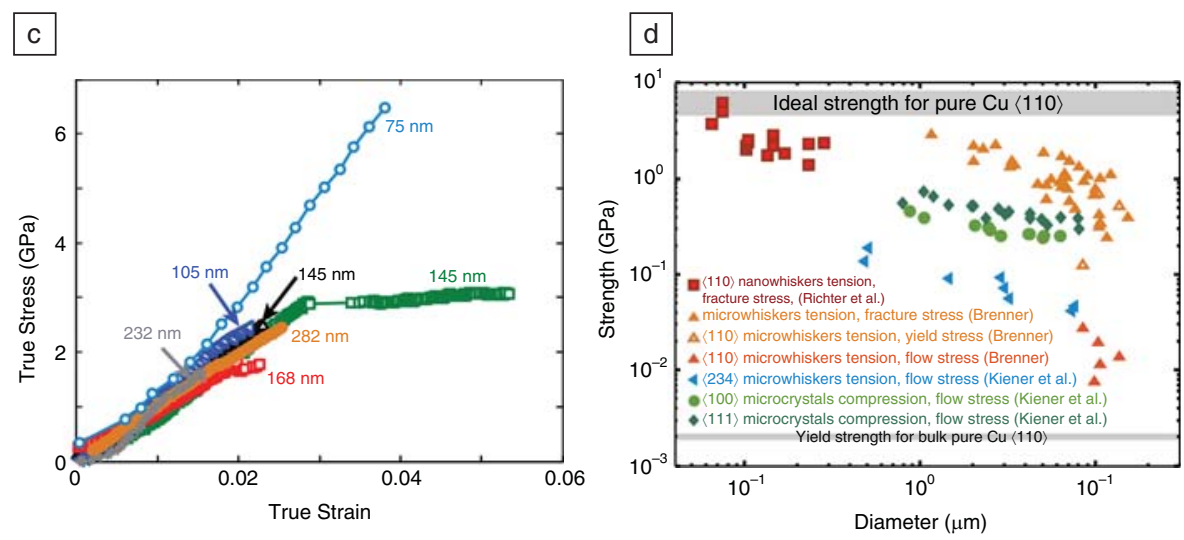

Figure 5. Quantitative in situ tensile testing of individual $\langle 110\rangle$ Cu nanowhiskers grown in ultrahigh vacuum molecular beam epitaxy conditions. (a) Scanning electron microscopy micrograph of a nanowire mechanical testing configuration. (b) Fracture morphologies showing both shear and cleavage failure. (c) Stress-strain curves for different effective diameters of nanowhiskers, as given by the numbered labels. (d) Size-dependent strength compared to reported values of strength of pure Cu show fracture strengths on the order of the computed ideal strength, which are attributed to the lack of pre-existing defects. ${ }^{63}$ 
manner (Figure 5b) with limited amounts of inelastic response measured from stressstrain curves (Figure 5c). These recent in situ instrumented tensile tests of defect-free $\mathrm{Cu}$ nanowhiskers complement the experiments of Bei ${ }^{36}$ and Brenner, $, 57,65$ as they show a departure from pillar behavior and give strengths at or near the ideal strength.

Large-scale plasticity does not occur in these whiskers, as supported by the lack of plastic flow in the stress-strain curves, as well as postmortem TEM characterization showing an absence of stored dislocations in fractured whiskers. These results differ from those obtained from smallscale experiments on fcc single crystals (Figure 5d) of presumably lower crystal quality, which demonstrate smaller strengths and a clear size effect ("smaller is stronger").

From these three recent examples of in situ experiments (dislocation accumulation with strain gradients, constant dislocation density in submicron crystal, and near theoretical strength in perfect whiskers), the emerging picture suggests that the presence or absence of pre-existing defects in small volumes greatly contributes to the measured mechanical response of the material and points to the need for thorough nanostructural characterization to accurately predict deformation and strength. Investigations yielding direct evidence of the strength-and rate-controlling deformation mechanisms in these and other ultra-strength nanomaterials represent a promising avenue for future inquiries.

\section{Polycrystals}

The absence of dislocations in smaller nanocrystals also has been used as a justification for their very high strength. However, they also do not reach stresses as high as perfect crystals, and GB-mediated plasticity often has been pointed to as the governing process in nanocrystals. At variance with dislocation-based plasticity, comparatively little is known about the shear produced by the migration of a GB, and, here again, in situ TEM experiments offer significant insight.

Figure 6 illustrates an in situ TEM experiment in which a line of GBs becomes mobile under stress. Between Figure $6 a$ and $6 \mathrm{~b}$, the GB has moved to the right and to the upper side of the picture, erasing grains G2 and G4. In Figure 6c, some particles at the surface (red squares) serve as references to measure the local shear noted $\mathrm{S}_{1}$ to $\mathrm{S}_{6}$. The black and white contrast, visible in red squares in Figure 6c, is directly related to the shear (red arrows) produced by the motion of the GB. As expected, the shear is larger when farther away from the moving GB. In the reference grain (G0), the particles in the black squares are fixed

a

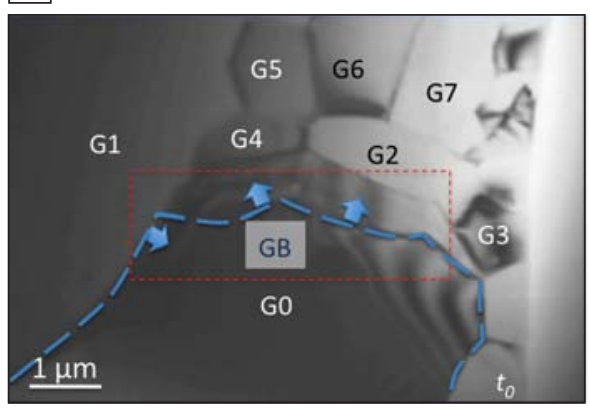

$\mathrm{b}$

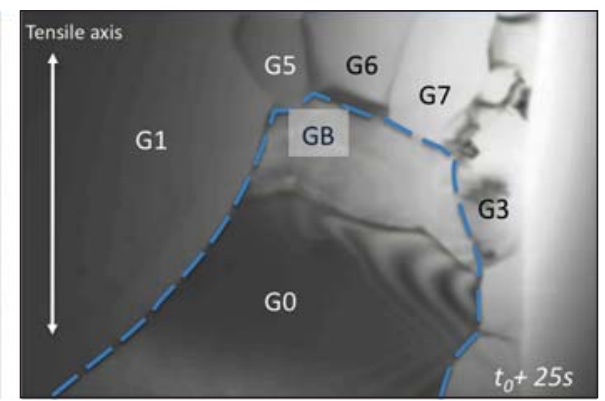

C

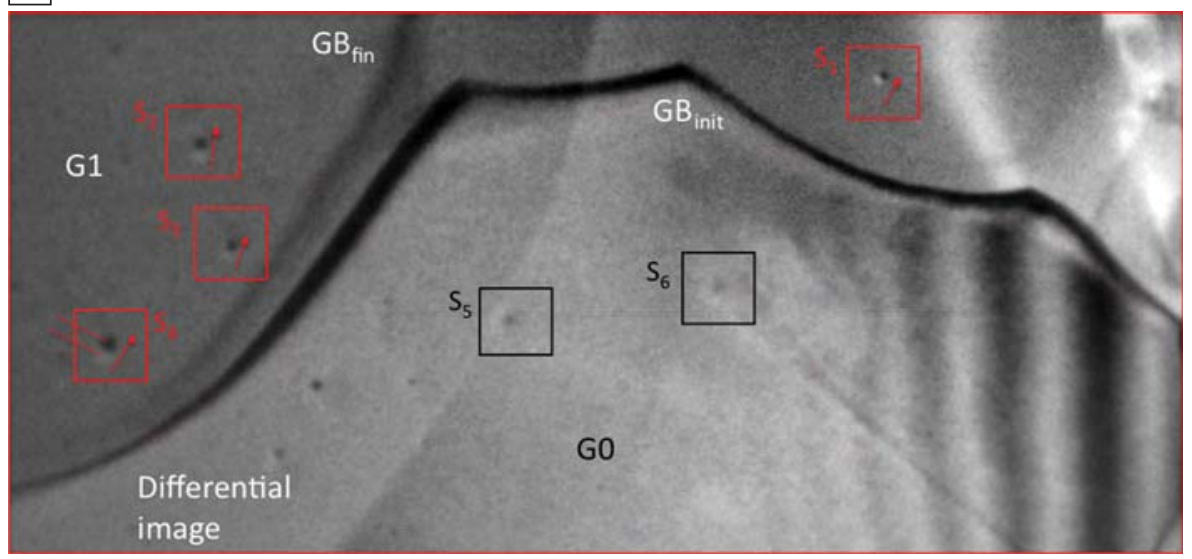

Figure 6. Grain-boundary (GB) migration-induced plasticity observed during in situ transmission electron microscopy straining of ultrafine-grain $\mathrm{Al}$ at $300^{\circ} \mathrm{C}(\mathrm{a}-\mathrm{b})$ (tensile axis is vertical). The blue arrows indicate the migration direction of the GB. Grains G1 to G7 are marked. Note the disappearance of grains G2 and G4 in (b). (c) Image correlation-based measurement of induced strain and subsequent determination of shear-migration coupling factor. The particles at the surface (red squares) act as references to measure the local shear noted $S_{1}$ to $S_{6}$. In the reference grain(G0), the particles in the black squares are fixed because they have not been crossed by a GB.

because they have not been crossed by a GB. The so-called $\beta$ factor, which is equal to the shear divided by the migration distance, characterizes the shear-coupling plasticity due to a migrating GB. In such experiments, all quantities are known, $\beta$, along with each grain orientation and therefore each GB character. It is thus possible to directly confront the GB-based plasticity theories, such as the one from Cahn and Mishin. ${ }^{66,67}$ In fact, the $\beta$ factors measured in these experiments ${ }^{53}$ were systematically much lower than those predicted by the Cahn model. Based on these in situ TEM experiments, a new theory has been proposed that is able to generate a larger data set of coupling factors, even for noncoincident GBs. ${ }^{52}$

\section{Summary and Outlook}

In situ electron microscopy is a rapidly evolving field that benefits from technological advancement of instrumentation (e.g., focused-ion-beam scanning electron microscopy platforms, fast charge-coupled device cameras, new piezoelectric-based transmission electron microscopy [TEM] holders, aberration-corrected TEMs) and improved quality of testing specimens, especially at the micron and nanometer scale. To investigate the mechanical properties of materials in very small dimensions, these techniques have established themselves as mandatory steps because they provide data measured at the appropriate length scale, along with simultaneous dynamic observations that are essential to understanding the underlying physical mechanisms. In this article, we have illustrated this virtuous combination in several cases, ranging from microbending beams to nano-sized crystals and whiskers. Developments allowing for quantification of data collected by in situ mechanical testing, such as digital image correlation, are rapidly evolving to exploit dynamical images produced by in situ electron microscopy and could be successfully 
applied to vastly unexplored fields such as grain boundary-mediated plasticity.

\section{Acknowledgments}

M.L. is indebted to F. Mompiou, D. Caillard, G. Dehm, S.H. Oh, and D. Kiener, who greatly contributed to the presented transmission electron microscopy results. D.S.G. is grateful for stimulating discussions with C. Eberl and M. Uchic and for financial support from the School of Engineering and Applied Sciences at the University of Pennsylvania.

\section{References}

1. Z. Budrovic, H. Van Swygenhoven, P.M. Derlet, S. Van Petegem, B. Schmitt, Science 304 (5668), 273 (2004).

2. R. Maass, S. Van Petegem, D. Grolimund, H. Van Swygenhoven, D. Kiener, G. Dehm, Appl. Phys. Lett. 92 (7), 071905 (2008).

3. J.S. Kim, T. LaGrange, B.W. Reed, M.L. Taheri, M.R. Armstrong, W.E. King, N.D. Browning, G.H. Campbell, Science 321 (5895), 1472 (2008).

4. D. Shorokhov, A.H. Zewail, Phys. Chem. Chem. Phys. 10 (20), 2879 (2008).

5. J.K. Deuschle, G. Buerki, H.M. Deuschle, S. Enders, J. Michler, E. Arzt, Acta Mater. 56 (16), 4390 (2008).

6. K.A. Rzepiejewska-Malyska, G. Buerki, J. Michler, R.C. Major, E. Cyrankowski, S.A.S. Asif, O.L. Warren, J. Mater. Res. 23 (7), 1973 (2008). 7. N. Gane, F.P. Bowden, J. Appl. Phys. 39 (3), 1432 (1968).

8. N. Gane, Proc. R. Soc. London, Ser. A 317 (1530), 367 (1970).

9. D.J. Dingley, in Proc. 3rd Annual SEM Symposium IIT, Res. Inst., Chicago, IL (1970).

10. N.A. Fleck, G.M. Muller, M.F. Ashby, J.W. Hutchinson, Acta Mater. 42, 475 (1994).

11. J.S. Stolken, A.G. Evans, Acta Mater. 46, 5109 (1998).

12. M.A. Haque, M.T.A. Saif, Acta Mater. 51 3053 (2003).

13. C. Motz, T. Schöberl, R. Pippan, Acta Mater 53 (15), 4269 (2005)

14. S. Wurster, C. Motz, M. Jenko, R. Pippan, Adv. Eng. Mater. 12, 61 (2009).

15. J. Silcox, M.J. Whelan, Philos. Mag. 5, 1 (1960). 16. F. Louchet, L.P. Kubin, D. Vesely, Philos. Mag. A. 39 (4), 433 (1979)

17. A. Couret, J. Crestou, S. Farenc, G. Molenat, N. Clément, A. Coujou, D. Caillard, Microsc Microanal. Microstruct. 4, 153 (1993).
18. A. Jacques, A. George, M. Polcarova, J. Bradler, Nucl. Instrum. Methods Phys. Res., Sect. B 200, 261 (2003)

19. G. Vanderschaeve, D. Caillard, Mater. Sci. Eng., A 462 (1-2), 418 (2007).

20. F. Mompiou, D. Caillard, M. Feuerbacher, Philos. Mag. A. 84 (25-26), 2777 (2004).

21. M. Legros, A. Couret, D. Caillard, Philos. Mag. A 73 (1), 81 (1996).

22. M. Legros, Y. Minonishi, D. Caillard, Philos. Mag. A 76 (5), 1013 (1997).

23. M. Legros, N. Clement, P. Caron, A. Coujou, Mater. Sci. Eng., A 337 (1-2), 160 (2002).

24. S. Farenc, A. Coujou, A. Couret, Philos. Mag. 67 (11), 127 (1993).

25. S.H. Oh, M. Legros, D. Kiener, P. Gruber, G. Dehm, Acta Mater. 55 (16), 5558 (2007).

26. M. Legros, D.S. Gianola, K.J. Hemker, Acta Mater. 56, 3380 (2008).

27. D. Caillard, Philos. Mag. Lett. 89 (8), 517 (2009). 28. M.D. Uchic, D.M. Dimiduk, Mater. Sci. Eng., A 400-401 (1-2 Suppl.), 268 (2005).

29. M.D. Uchic, D.M. Dimiduk, J.N. Florando, W.D. Nix, Science 305 (5686), 986 (2004).

30. J.R. Greer, C.R. Weinberger, W. Cai, Mater. Sci. Eng., A 493 (1-2), 21 (2008).

31. J. Greer, J.-Y. Kim, M. Burek, JOM 61 (12), 19 (2009).

32. D. Kiener, W. Grosinger, G. Dehm, R. Pippan, Acta Mater. 56 (3), 580 (2008)

33. Z.W. Shan, R.K. Mishra, S.A.S. Asif, O.L. Warren, A.M. Minor, Nat. Mater. 7 (2), 115 (2008). 34. H. Bei, S. Shim, M.K. Miller, G.M. Pharr, E.P. George, Appl. Phys. Lett. 91 (11), 111915 (2007).

35. S. Shim, H. Bei, M.K. Miller, G.M. Pharr, E.P. George, Acta Mater. 57 (2), 503 (2009).

36. H. Bei, S. Shim, E.P. George, M.K. Miller, E.G. Herbert, G.M. Pharr, Scr. Mater. 57 (5), 397 (2007)

37. D. Zhang, J.-M. Breguet, R. Clavel, L. Phillippe, I. Utke, J. Michler, Nanotechnology 20, 36 (2009)

38. F. Östlund, K. Rzepiejewska-Malyska, K. Leifer, L.M. Hale, Y. Tang, R. Ballarini, W.W. Gerberich, J. Michler, Adv. Funct. Mater. 19 (15), 2439 (2009)

39. M. Legros, M. Cabié, D.S. Gianola, Microsc. Res. Tech. 72 (3), 270 (2009)

40. M. Jin, A.M. Minor, E.A. Stach, J.J.W. Morris, Acta Mater. 52 (18), 5381 (2004).

41. J.H. Han, M.T.A. Saif, Rev. Sci. Instrum. 77, 4 (2006).

42. M.A. Haque, M.T.A. Saif, J. Mater. Res. 20 (7), 1769 (2005)

43. J. Rajagopalan, J.H. Han, M.T.A. Saif, Science 315, 1831 (March 2007).
44. J. Rajagopalan, J.H. Han, M.T.A. Saif, Scr. Mater. 59, 734 (2008).

45. M. Legros, K.J. Hemker, A. Gouldstone, S. Suresh, R.M. Keller-Flaig, E. Arzt, Acta Mater. 50 (13), 3435 (2002)

46. M. Legros, Relaxation plastique des couches métalliques par dislocations et défauts étendus, in Contraintes mécaniques en micro, nano et optoélectronique (Traité EGEM, série Electronique et microélectronique), M. Mouis, Ed. (Hermes Science Publications, Paris, 2006).

47. Y. Zhu, A. Corigliano, H.D. Espinosa, J. Micromech. Microeng. 16, 242 (2006).

48. A.M. Minor, S.A.S. Asif, Z.W. Shan, E.A. Stach, E. Cyrankowski, T.J. Wyrobek, O.L. Warren, Nat. Mater. 5 (9), 697 (2006).

49. T. Zhu, J. Li, S. Ogata, S. Yip, MRS Bull. 34 (3), 167 (2009)

50. T.C. Chu, W.F. Ranson, M.A. Sutton, W.H. Peters, Exp. Mech. 25 (3), 232 (1985).

51. M.A. Sutton, N. Li, D.C. Joy, A.P. Reynolds, X. Li, Exp. Mech. 47 (6), 775 (2007).

52. D. Caillard, F. Mompiou, M. Legros, Acta Mater. 57 (8), 2390 (2009).

53. F. Mompiou, D. Caillard, M. Legros, Acta Mater. 57 (7), 2198 (2009).

54. M.J. Hÿtch, J.-L. Putaux, J.-M. Pénisson, Nature 425, 270 (2003).

55. M.J. Hÿtch, F. Houdellier, F. Hüe, E. Snoeck, Nature 453, 1086 (2008).

56. M. Uchic, P. Shade, D. Dimiduk, JOM 61 (3), 36 (2009).

57. S.S. Brenner, J. Appl. Phys. 27 (12), 1484 (1956) 58. H. Bei, Y.F. Gao, S. Shim, E.P. George, G.M. Pharr, Phys. Rev. B: Condens. Matter 77 (6), 060103 (2008).

59. D.M. Norfleet, D.M. Dimiduk, S.J. Polasik, M.D. Uchic, M.J. Mills, Acta Mater. 56 (13), 2988 (2008).

60. C. Motz, D. Weygand, J. Senger, P. Gumbsch, Acta Mater. 56 (9), 1942 (2008).

61. G. Dehm, M. Legros, B. Heiland, J. Mater. Sci. 41, 4484 (2006).

62. S.H. Oh, M. Legros, D. Kiener, G. Dehm, Nat. Mater. 8, 95 (2009).

63. G. Richter, K. Hillerich, D.S. Gianola, R. Mönig O. Kraft, C.A. Volkert, Nano Lett. 9 (8), 3048 (2009). 64. S. Ogata, J. Li, N. Hirosaki, Y. Shibutani, S. Yip, Phys. Rev. B: Condens. Matter 70 (10), (2004). 65. S.S. Brenner, J. Appl. Phys. 28 (9), 1023 (1957). 66. J.W. Cahn, J.E. Taylor, Acta Mater. 52 (16), 4887 (2004).

67. J.W. Cahn, Y. Mishin, A. Suzuki, Acta Mater. 54 (19), 4953 (2006).

68. D.S. Gianola, C. Eberl, JOM 61 (3), 24 (2009).

\section{In situ TEM Systems \\ Nanofactory Instruments provides different}

Application: SPM technologies as STM, AFM and Nanolndentation for in situ TEM.

Key Feature:

Nano and microforce measurements, conductivity measurements.

\section{nano FACTORY}

Contact information: info@nanofactory.com Chalmers Science Park, SE-41288, Göteborg, Sweden Phone: +46 317190720 Fax: +46 31165985 www.nanofactory.com 
Bruker AXS

50 years of

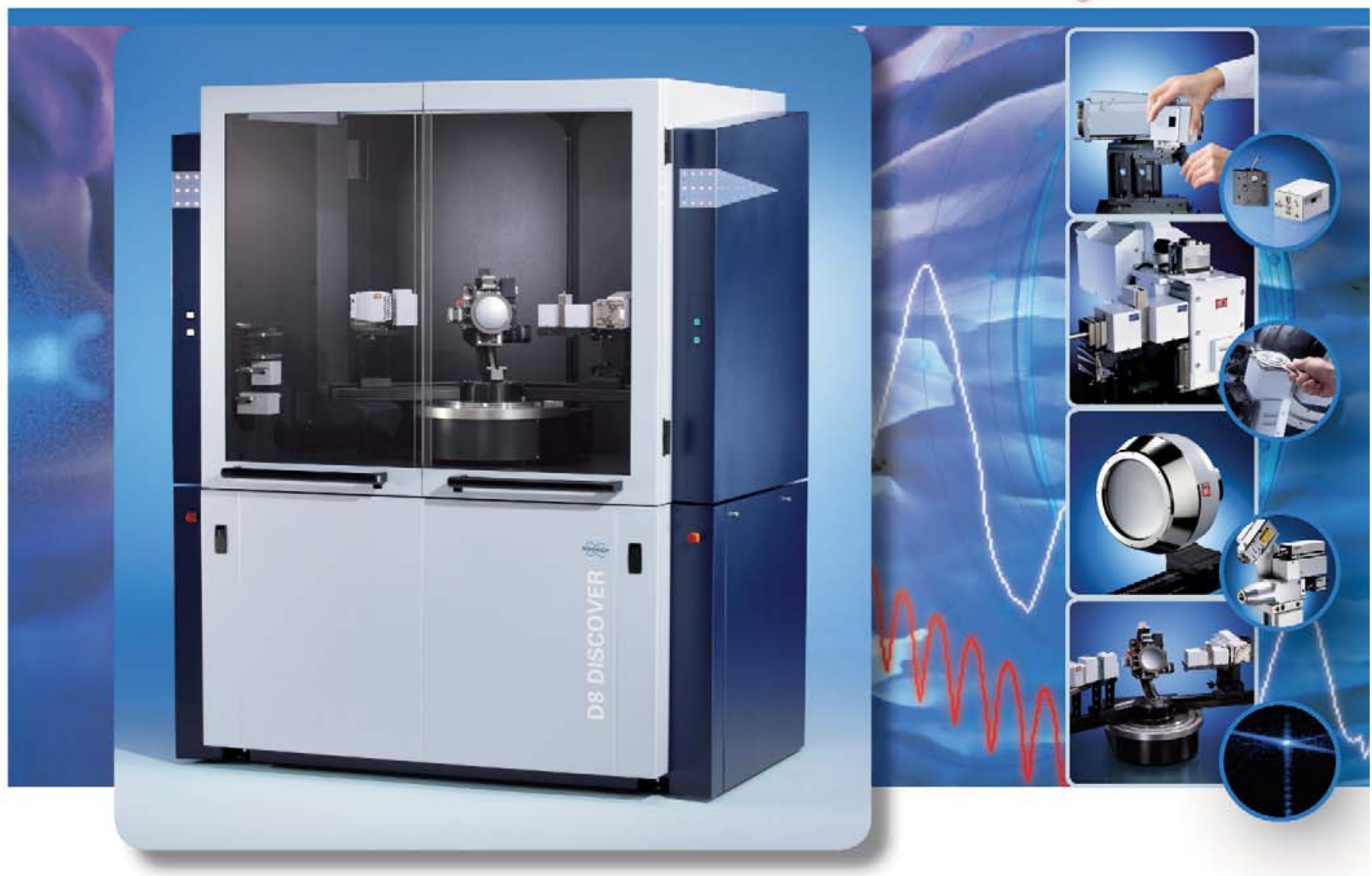

\section{The new D8 DISCOVER with DAVINCI}

\section{- Move up to the $\mathrm{XRD}^{2}$ dimension in X-ray diffraction}

- DAVINCI.SNAP-LOCK \& DAVINCI.MODE: alignment-free component change with real-time recognition

- TURBO X-RAY SOURCE: boost intensity for point, line or micro focus applications

- TWIST-TUBE: fast and easy switching from line to point focus

- VÅNTEC-500: add the extra dimension of XRD22

- PATHFINDER optics: motorized switching between high-resolution and high-intensity beam paths

www.bruker-axs.com

think forward 